\title{
Association between polycystic ovary syndrome and risk of attention-deficit/hyperactivity disorder in offspring: a meta-analysis
}

To the editor: We read with great interest a meta-analysis by Maleki et al. ${ }^{1)}$ on the association between prenatal excess of androgen exposure in the offspring of mothers with polycystic ovary syndrome (PCOS) and risk of attention-deficit/hyperactivity disorder (ADHD) in them. They found that maternal PCOS increases the offspring risk of ADHD. However, we have to point out a deficiency of this study, we noted that 1 of 6 articles that were included in this study did not meet the eligibility criteria as it's object was different. ${ }^{2}{ }^{2}$ Hergüner et al..2) aimed to compare ADHD symptoms between women with PCOS and controls. This deficiency may introduce bias and lead to a false conclusion.

\section{Hadith Rastad ${ }^{1}$, Mahnaz Seifi Alan²}

${ }^{1}$ Non-communicable Diseases Research Center, Alborz University of Medical Sciences, Karaj, Iran; ${ }^{2}$ Cardiovascular Research Center, Alborz University of Medical Sciences, Karaj, Iran

Corresponding author: Mahnaz Seifi Alan

Cardiovascular Research Center, Alborz University of Medical Sciences, Karaj, Iran

凶Email:m_seifi2012@yahoo.com

https://orcid.org/0000-0003-1882-5951

Conflicts of interest: No potential conflict of interest relevant to this article was reported.

Funding: This letter received no specific grant from any funding agency in the public, commercial, or not-for-profit sectors.

1. Maleki A, Bashirian S, Soltanian AZ, Jenabi E, Farhadinasab A. Association between polycystic ovary syndrome and risk of attention-deficit/ hyperactivity disorder in offspring: a meta-analysis. Clin Exper Pediatr 2021;37:716-20.

2. Hergüner S, Harmancı $\mathrm{H}$, Toy $\mathrm{H}$. Attention deficit-hyperactivity disorder symptoms in women with polycystic ovary syndrome. Int J Psychiatry Med 2015;50:317-25.

https://doi.org/10.3345/cep.2021.01298.r1
The authors reply: We appreciate your opinion and your suggestions, and I'd like to reply to the points that you mentioned. We in this meta-analysis assessed the association between polycystic ovary syndrome (PCOS) and risk of attention-deficit/ hyperactivity disorder (ADHD) in offspring compared with women without PCOS. ${ }^{1)}$ Hergüner et al. ${ }^{2)}$ aimed to compare ADHD symptoms between women with PCOS and controls. However, Hergüner et al..$^{2)}$ measured ADHD symptoms based on 18 items on Diagnostic and Statistical Manual of Mental Disorders, 4th edition (DSM-IV) criteria for ADHD. DSM-IV is a diagnostic critera for ADHD.,3) Therefore, the symptoms of ADHD based on DSM-IV criteria indicate the diagnosis of ADHD. On the other, there was a significant association between PCOS and risk ADHD in 5 of 6 included articles. Therefore, it cannot introduce bias and a false conclusion.

\section{Ensiyeh Jenabi}

Autism Spectrum Disorders Research Center, Hamadan University of Medical Sciences, Hamadan, Iran, Hamadan, Iran

Corresponding author: Ensiyeh Jenabi

Autism Spectrum Disorders Research Center, Hamadan University of Medical Sciences, Hamadan, Iran, Hamadan, Iran

凶Email: en.jenabi@yahoo.com

https://orcid.org/0000-0002-4536-0814

Conflicts of interest: No potential conflict of interest relevant to this article was reported.

Funding: This letter received no specific grant from any funding agency in the public, commercial, or not-for-profit sectors.

1. Kosidou K, Dalman C, Widman L, Arver S, Lee BK, Magnusson C, et al. Maternal polycystic ovary syndrome and risk for attention-deficit/ hyperactivity disorder in the offspring. Biol Psychiatry 2017;82:651-9.

2. Hergüner $S$, Harmanc $\mathrm{H}$, Toy $\mathrm{H}$. Attention deficit-hyperactivity disorder symptoms in women with polycystic ovary syndrome. Int J Psychiatry Med 2015;50:317-25.

3. Epstein J, Johnson DE, Conners CK. Conners' adult ADHD diagnostic interview for DSM-IV ${ }^{\mathrm{TM}}\left(\mathrm{CAADID}^{\mathrm{TM}}\right)$. North Tonawanda (NY): MultiHealth Systems Inc.; 2001.

4. McBurnett K, Lahey BB, Pfiffner LJ. Diagnosis of attention deficit disorders in DSM-IV: Scientific basis and implications for education. Except Child 1993;60:108-17.

https://doi.org/10.3345/cep.2021.01298.r2 